\title{
Electromagnetically induced transparency inside the laser cavity: Switch between first-order and second-order phase transitions
}

\author{
Qingqing Sun, ${ }^{1,2}$ M. Selim Shahriar, ${ }^{3}$ and M. Suhail Zubairy ${ }^{1,2}$ \\ ${ }^{1}$ Department of Physics and Institute of Quantum Studies, Texas A\&M University, College Station, Texas 77843-4242, USA \\ ${ }^{2}$ Texas A\&M University at Qatar, Education City, P.O. Box 23874, Doha, Qatar \\ ${ }^{3}$ Department of Electrical Engineering and Computer Science, Northwestern University, Evanston, Illinois 60208-3118, USA
}

(Received 22 January 2008; published 2 July 2008)

\begin{abstract}
We investigate the effect of electromagnetically induced transparency inside a laser cavity. By changing the intensity of an external drive field, we can control the absorption to the laser field. A semiclassical analysis shows that the system undergoes a switch between first-order and second-order phase transitions. Around the tricritical point there could be a second-order phase transition followed by a first-order phase transition.
\end{abstract}

DOI: 10.1103/PhysRevA.78.013805

PACS number(s): 42.50.Gy, 68.35.Rh

\section{INTRODUCTION}

Lasing is a cooperative phenomenon of many atoms in a cavity. Each atom has a dipole induced by the field from all the other atoms; in turn its dipole also contributes to the field, which can be uniformly treated by the mean field theory. Although laser is a nonequilibrium system, the formal similarity enables the analogy of its near-threshold behavior to second-order phase transitions in the equilibrium systems [1-3]. For example, in the analogy to the ferromagnetic phase transition, the electric field corresponds to the magnetization $M$, the unsaturated population inversion corresponds to the temperature, and an injected field corresponds to the external magnetic field $H$. The measurement of the laser "coexistence curve" and "susceptibility" below threshold confirmed this analogy quantitatively [4]. As a completion of this analogy, Gatti and Lugiato showed that the correlation length of a degenerate optical parametric oscillator diverges when it approaches the threshold [5].

Once the second order phase transition analogy was established, people started looking for similar analogy in first order. Examples include the laser with a saturable absorber (LSA) [6-8] and the dye laser [9]. Here we will concentrate on the LSA problem. The saturable absorber is inside the laser cavity. The absorption saturation changes the nature of the system. So the laser output can have two stable values and leads to bistability, hysteresis, etc. Experiments have been done in many systems, e.g., He:Ne laser [10], $\mathrm{CO}_{2}$ laser with $\mathrm{SF}_{6}$ absorber [11], and $\mathrm{N}_{2} \mathrm{O}$ laser with $\mathrm{NH}_{3}$ absorber [12]. In theory, Mandel and co-workers treated the problem analytically in both semiclassical and quantum theories [13]. In their model both the active cell and the absorber cell contain two-level atoms, interacting with a single cavity mode. The relative saturability and population inversion of the two cells determine the number of roots. Hysteresis cycle is obtained when two stable roots are available. The quantum theory based on Fokker-Planck equation gives the field fluctuation and linewidth across the threshold. It is shown that the finite fluctuation increases drastically when approaching the threshold, and then goes to zero above the threshold. The linewidth also has a sharp narrowing. In a related paper [14] they showed that the inclusion of higher order derivative terms reduces the width of the transition region, making the transition threshold much sharper. This model has also been used to study the time-dependent behavior [15]. Small amplitude harmonically modulated intensity and pulses corresponding to the passive $Q$ switching are found to be the stable solutions. Roy gave the photon distribution in this system and showed the similarity to the dye laser [16].

As another concept in the phase transition theory, a tricritical point is the joint of a first-order phase transition line to a second-order line in the parameter space. Scott pointed out the existence of a tricritical point in his LSA model [17]. Mortazavi and Singh measured the tricritical behavior by changing the discharge current of the absorber cell [18]. Later they found that the intensity fluctuation undergoes a qualitative change through the tricritical point [25]. For a second-order phase transition, the fluctuation decreases from the thermal value to zero with increasing excitation, while for a first-order phase transition it first increases to a superthermal value and then decreases to zero.

Systems with multistability and multicritical points have also been explored [19-21]. It is shown that when the potential has higher order terms or when a multimode laser introduces more order parameters, there could be multistability and multicritical points.

Three-level systems have also been used to model the absorber. One example is the two-photon absorber [22], in which the middle level is assumed to be far from resonance so that the system is very similar to a two-level system. Agrawal considered the case of a $\Lambda$ system [23]. The laser frequency is assumed to lie midway between the ground state sublevels. The phase transition is determined by the detuning, which can be controlled by a static electric or magnetic field. He predicted the switch between first-order and second-order phase transitions similar to our result, although the mechanism is different.

We propose a system based on electromagnetically induced transparency (EIT) [24]. The laser field resonant with one transition should have been absorbed. But at the presence of another drive field resonant with another transition with a common level, the coherence effect renders transparency for the laser field. This property intrigues our interest in the possible application into a LSA problem. Since the drive field can control the absorption of the probe field, it might simulate a system of laser with or without a saturable ab- 
sorber. As a result, we would be able to switch between first-order and second-order phase transitions by simply adjusting the drive field intensity.

\section{FREE ENERGY FOR A LASER WITH OR WITHOUT A SATURABLE ABSORBER}

From Landau's theory the Gibbs free energy close to a critical point can be expanded into even powers of a displacement parameter $x$,

$$
G(x)=C_{2} x^{2}+C_{4} x^{4}+C_{6} x^{6}+\ldots,
$$

where the coefficient $C_{2}$ is linear to the difference between the reservoir variable and its critical value. The phase transition is second order if $C_{4}$ is positive, while first order if $C_{4}$ is negative.

A potential similar to the Gibbs free energy can be defined for a laser [1]. The displacement parameter is the electric field $\mathcal{E}$. One can obtain a Fokker-Planck equation from the laser theory and then apply it to the electric field to get an equation of motion for the expectation value. The potential can then be obtained by integration from

$$
\dot{\mathcal{E}}=-\frac{\partial G(\mathcal{E})}{\partial \mathcal{E}} .
$$

For a laser without an absorber, the equation of motion is well known as

$$
\dot{\mathcal{E}}=\frac{1}{2}\left[(\mathcal{A}-\mathcal{C}) \mathcal{E}-\mathcal{B} \mathcal{E}^{3}\right],
$$

where $\mathcal{A}$ is the unsaturated gain in the active medium, $\mathcal{B}$ is the saturation parameter, and $\mathcal{C}$ is the cavity loss. Both $\mathcal{A}$ and $\mathcal{B}$ are proportional to the population inversion $\sigma$. A simple integration gives $C_{4}=\mathcal{B} / 8$ which is positive. So it is a second-order phase transition. The stable value of $\mathcal{E}$ changes continuously as one move across the critical point.

For a laser with a saturable absorber inside the cavity, the equation of motion can be modified as [8]

$$
\dot{\mathcal{E}}=\frac{1}{2}\left[\left(\mathcal{A}-\mathcal{C}-\frac{S}{1+\mathcal{E}^{2} / I_{s}}\right) \mathcal{E}-\mathcal{B E}^{3}\right],
$$

where $S$ is the linear absorption from the saturable absorber and $I_{S}$ is the saturation intensity. After integration the potential has a logarithmic term coming from the saturable absorber. Power series expansion gives

$$
C_{4}=\frac{1}{8}\left(\mathcal{B}-\frac{S}{I_{S}}\right)
$$

So if the saturation intensity of the absorber is small, $I_{s}$ $\leq S / \mathcal{B}$, the phase transition would be first order. The field has a discontinuous jump from zero to some finite value when crossing the critical point.

\section{DERIVATION AND DISCUSSION}

The setup for our system is shown in Fig. 1. We use a unidirectional ring laser cavity and assume both kinds of

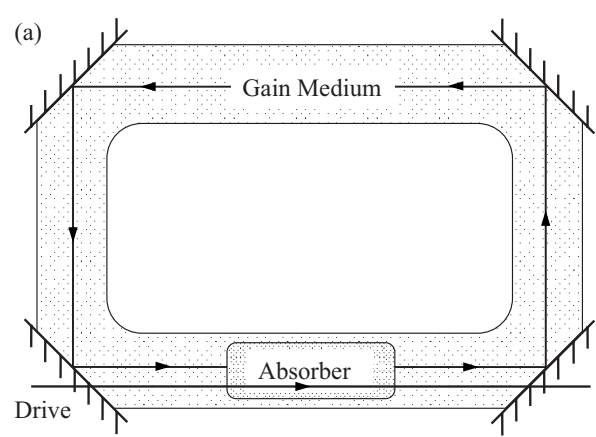

(b)

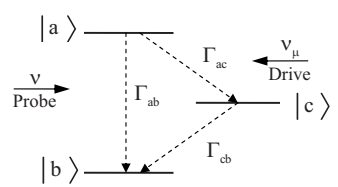

FIG. 1. (a) The setup of the system. The absorber cell filled with three-level atoms is put inside the unidirectional ring laser cavity. (b) The level structure for the three-level atoms.

atoms are homogenously broadened. The incoherent pumping in the gain medium provides population inversion for the active atoms, generating a laser field as the probe field for the $\Lambda$-type three-level atoms in the absorber cell. This probe field with frequency $\nu$ interacts with the transition $|a\rangle \leftrightarrow|b\rangle$, while the external drive field with frequency $\nu_{\mu}$ interacts with the transition $|a\rangle \leftrightarrow|c\rangle$. To avoid drive field recycling we assume the mirrors to be transparent to the drive field.

For a closed system, the density matrix equations are

$$
\begin{aligned}
\dot{\rho}_{b b}= & \Gamma_{a b} \rho_{a a}+\Gamma_{c b} \rho_{c c}-\frac{i}{2 \hbar}\left(\wp_{a b} \mathcal{E} e^{-i \nu t} \rho_{b a}-\text { c.c. }\right), \\
\dot{\rho}_{c c}= & \Gamma_{a c} \rho_{a a}-\Gamma_{c b} \rho_{c c}-\frac{i}{2}\left(\Omega_{\mu} e^{-i \phi_{\mu}} e^{-i \nu_{\mu} t} \rho_{c a}-\text { c.c. }\right), \\
\dot{\rho}_{a a}= & -\left(\Gamma_{a b}+\Gamma_{a c}\right) \rho_{a a}+\frac{i}{2 \hbar}\left(\wp_{a b} \mathcal{E} e^{-i \nu t} \rho_{b a}-\text { c.c. }\right) \\
& +\frac{i}{2}\left(\Omega_{\mu} e^{\left.-i \phi_{\mu} e^{-i \nu} \mu^{t} \rho_{c a}-c . c .\right),}\right. \\
\dot{\rho}_{a b}= & -\left(i \omega_{a b}+\gamma_{a b}\right) \rho_{a b}-\frac{i \wp_{a b} \mathcal{E}}{2 \hbar} e^{-i \nu t}\left(\rho_{a a}-\rho_{b b}\right) \\
& +\frac{i}{2} \Omega_{\mu} e^{-i \phi_{\mu} e^{-i \nu_{\mu} t} \rho_{c b},}
\end{aligned}
$$

$$
\dot{\rho}_{c b}=-\left(i \omega_{c b}+\gamma_{c b}\right) \rho_{c b}-\frac{i \wp_{a b} \mathcal{E}}{2 \hbar} e^{-i \nu t} \rho_{c a}+\frac{i}{2} \Omega_{\mu} e^{i \phi_{\mu}} e^{i \nu_{\mu} t} \rho_{a b},
$$




$$
\begin{aligned}
\dot{\rho}_{c a}= & -\left(i \omega_{c a}+\gamma_{c a}\right) \rho_{c a}+\frac{i}{2} \Omega_{\mu} e^{i \phi_{\mu}} e^{i \nu_{\mu} t}\left(\rho_{a a}-\rho_{c c}\right) \\
& -\frac{i \wp_{b a} \mathcal{E}^{*}}{2 \hbar} e^{i \nu t} \rho_{c b},
\end{aligned}
$$

where $\wp_{a b}$ is the electric dipole moment, $\mathcal{E}$ is the probe field produced by the active atoms, $\Omega_{\mu}$ is the drive Rabi frequency, $\Gamma$ and $\gamma$ are the population relaxation rates and dipole decay rates, respectively.

For simplicity, we assume both the drive field and the probe field to be resonant with their corresponding transitions. After transforming into the rotating frame,

$$
\rho_{a b}=\tilde{\rho}_{a b} e^{-i \omega_{a b} t}, \quad \rho_{c b}=\tilde{\rho}_{c b} e^{-i\left(\omega_{a b}-\nu_{\mu}\right) t}, \quad \rho_{c a}=\tilde{\rho}_{c a} e^{i \nu_{\mu} t} .
$$

Together with the relation $\rho_{a a}+\rho_{b b}+\rho_{c c}=1$ for the closed system, we can obtain the steady state solution. So the effective polarization from the absorber

$$
\begin{aligned}
\mathcal{P}= & 2 N_{a}^{e} \wp_{b a} \tilde{\rho}_{a b}=i N_{a}^{e} \frac{\left|\wp_{a b}\right|^{2} \mathcal{E}}{\hbar D}\left\{\left|\frac{\wp_{a b} \mathcal{E}}{2 \hbar}\right|^{2}\left(\Gamma_{a b}+\Gamma_{a c}\right) \Gamma_{c b}\right. \\
& \left.+\gamma_{c b}\left[\frac{\Omega_{\mu}^{2}}{2}\left(\Gamma_{a b}+\Gamma_{c b}\right)+\gamma_{c a}\left(\Gamma_{a b}+\Gamma_{a c}\right) \Gamma_{c b}\right]\right\},
\end{aligned}
$$

where the denominator

$$
\begin{aligned}
D= & \left|\frac{\wp_{a b} \mathcal{E}}{2 \hbar}\right|^{4}\left(2 \Gamma_{a c}+4 \Gamma_{c b}\right)+\left|\frac{\wp_{a b} \mathcal{E}}{2 \hbar}\right|^{2}\left[\gamma_{a b}\left(\Gamma_{a b}+\Gamma_{a c}\right) \Gamma_{c b}\right. \\
& \left.+\frac{\Omega_{\mu}^{2}}{2}\left(\Gamma_{a b}+\Gamma_{a c}-3 \Gamma_{c b}+6 \gamma_{c b}\right)+2 \gamma_{c b} \gamma_{c a}\left(\Gamma_{a c}+2 \Gamma_{c b}\right)\right] \\
& +\left(\frac{\Omega_{\mu}^{2}}{4}+\gamma_{a b} \gamma_{c b}\right)\left[\frac{\Omega_{\mu}^{2}}{2}\left(\Gamma_{a b}+\Gamma_{c b}\right)+\gamma_{c a}\left(\Gamma_{a b}+\Gamma_{a c}\right) \Gamma_{c b}\right],
\end{aligned}
$$

and $N_{a}^{e}$ is the effective number density for the three-level atoms. It can be related to the actual number density by $N_{a}^{e}$ $=N_{a} V_{a} / V_{\text {tot }}$. Here $V_{a}$ and $V_{\text {tot }}$ are the absorber volume and the total volume, respectively. Similarly we can define the effective number density for the gain medium $N_{g}^{e}$.

We assume the active atoms to be effectively two-level, which has the same level separation as $\omega_{a b}$ and the same dipole moment. With the polarization from the EIT medium, we can obtain the field equation of motion

$$
\dot{\mathcal{E}}=\frac{1}{2}\left[\left(\frac{\mathcal{A}}{1+\mathcal{E}^{2} / I_{s}}-\mathcal{C}\right) \mathcal{E}-\left(\frac{\nu}{\epsilon_{0}}\right) \operatorname{Im} \mathcal{P}\right],
$$

where $\mathcal{A}=\frac{\nu\left|\wp_{a b}\right|^{2} N_{g}^{e}}{\epsilon_{0} \hbar \gamma_{a b}}$ is the linear gain and $I_{s}=\frac{\hbar^{2} \Gamma_{a b}^{2}}{2\left|\wp_{a b}\right|^{2}}$ is the saturation intensity for the active atoms. After integration the coefficient of $\mathcal{E}^{4}$ term in $G(\mathcal{E})$ is

$$
C_{4}=\frac{1}{8} \frac{\mathcal{A}}{I_{s}}-\frac{\nu}{2} N_{a}^{e} \frac{\left|\wp_{a b}\right|^{4}}{4 \epsilon_{0} \hbar^{3}} \frac{\frac{\Omega_{\mu}^{2}}{4}\left[2 \gamma_{c b}\left(\Gamma_{a b}+\Gamma_{a c}-3 \Gamma_{c b}\right)-\left(\Gamma_{a b}+\Gamma_{a c}\right) \Gamma_{c b}+12 \gamma_{c b}^{2}\right]+2 \gamma_{c b}^{2} \gamma_{c a}\left(\Gamma_{a c}+2 \Gamma_{c b}\right)}{4\left(\frac{\Omega_{\mu}^{2}}{4}+\gamma_{a b} \gamma_{c b}\right)^{2}\left[\frac{\Omega_{\mu}^{2}}{2}\left(\Gamma_{a b}+\Gamma_{c b}\right)+\gamma_{c a}\left(\Gamma_{a b}+\Gamma_{a c}\right) \Gamma_{c b}\right]} .
$$

If there is no drive field, i.e., $\Omega_{\mu}=0$, it is simplified to,

$$
C_{4\left(\Omega_{\mu}=0\right)}=\frac{1}{8} \frac{\mathcal{A}}{I_{s}}-\frac{\nu}{2} N_{a}^{e} \frac{\left|\wp_{a b}\right|^{4}}{4 \epsilon_{0} \hbar^{3}} \frac{\Gamma_{a c}+2 \Gamma_{c b}}{2 \gamma_{a b}^{2}\left(\Gamma_{a b}+\Gamma_{a c}\right) \Gamma_{c b}} .
$$

For large enough $N_{a}^{e}$ we could have $C_{4\left(\Omega_{\mu}=0\right)}<0$. Then we increase the drive field intensity so that the second term of Eq. (16) has a smaller magnitude. Finally at some point $C_{4}$ $=0$. According to Ref. [8] this is the criterion between firstorder and second-order phase transitions.

In the limiting case of a perfect EIT system, the dephasing rate between the two lower levels $\gamma_{c b}=0$, which also requires $\Gamma_{c b}=0$. Substitute into Eqs. (16) and (17) and we find $C_{4\left(\Omega_{\mu} \neq 0\right)}=\mathcal{A} / 8 I_{s}$ while $C_{4\left(\Omega_{\mu}=0\right)} \rightarrow-\infty$. One might tend to say that the order of the phase transition just depends on whether we switch on the drive field or not. But the stable value of $\mathcal{E}$ would also go to infinity so it is a idealized situation. One can never achieve a perfect EIT.

For a finite $\gamma_{c b}$ we did the numerical calculation to find the steady state solutions for Eq. (15). These solutions will follow the change of $\mathcal{C}=\frac{\nu}{Q}$ where $Q$ is the quality factor of the cavity. As can be seen from Fig. 2, the probe intensity- $Q$ curve is single valued for a Rabi frequency above the threshold, while bistable for a Rabi frequency below the threshold. Those parts with a negative slope are unstable. So the switch between first-order and second-order phase transitions can be achieved by simply changing the drive Rabi frequency. For a small range near the threshold there are even three solutions. When the $Q$ factor increases, one can see a second-order phase transition followed by a first-order phase transition, as shown for $\Omega_{\mu}=0.1096 \mathrm{MHz}$.

The parameters we used are somehow extreme. The $Q$ factor and drive Rabi frequency are small. But the probe laser intensity is large, which is still acceptable since it is inside the cavity. There is no requirement for a small probe because we keep $\mathcal{E}$ to all orders in Eq. (13). An interesting thing is to compare the threshold value to the solution of $C_{4}=0$. Ideally they should be the same. But we found a small difference. This can be understood since the $C_{4}$ criterion is 

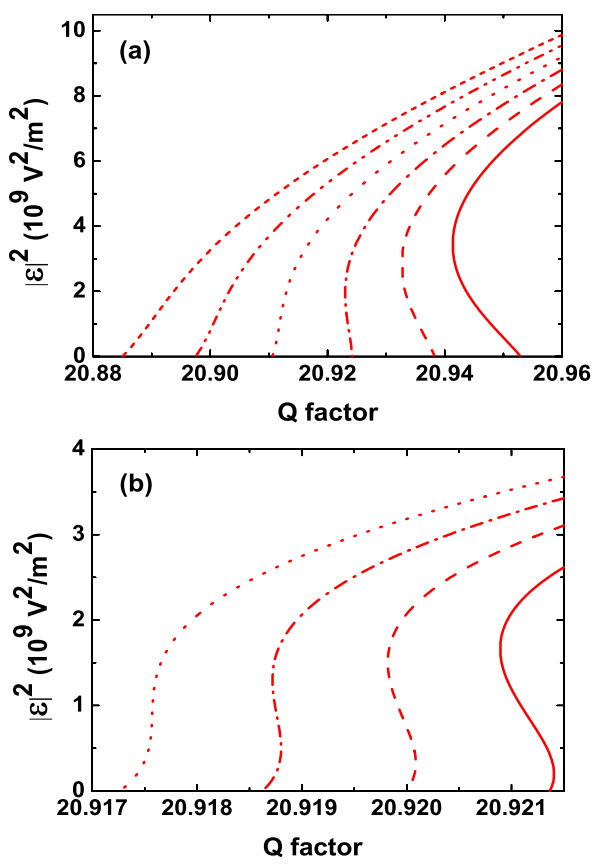

FIG. 2. (Color online) Intensity- $Q$ factor curves for different drive Rabi frequencies. (a) From right to left, $\Omega_{\mu}=0.100 \mathrm{MHz}$, $0.104 \mathrm{MHz}, 0.108 \mathrm{MHz}, 0.112 \mathrm{MHz}, 0.116 \mathrm{MHz}, 0.120 \mathrm{MHz}$. Clearly there is a switch between first-order and second-order phase transitions. (b) From right to left, $\Omega_{\mu}=0.1088 \mathrm{MHz}, 0.1092 \mathrm{MHz}$, $0.1096 \mathrm{MHz}, 0.1100 \mathrm{MHz}$. For a small range near the tricritical point, there could be three nonzero solutions. The other parameters are $\left|\wp_{a b}\right|=10^{-29} \mathrm{~m} \mathrm{C}, \quad \lambda=1 \mu \mathrm{m}, \quad N_{a}^{e}=4.5 \times 10^{16} \mathrm{~m}^{-3}, \quad N_{g}^{e}=1.28$ $\times 10^{18} \mathrm{~m}^{-3}$, and $\gamma_{c b}=1 \mathrm{kHz}$.

only good for small probe approximation, which does not apply quite well in this case.

The switch between first-order and second-order phase transition is a tricritical point in the phase space, as can be seen from the phase diagram Fig. 3. Such tricritical points have been predicted in the two-level atom saturable absorber case [18]. In that system the point is the termination of both lines, while here the first-order line goes beyond the tricritical point. So for a given drive Rabi frequency, we can ob-

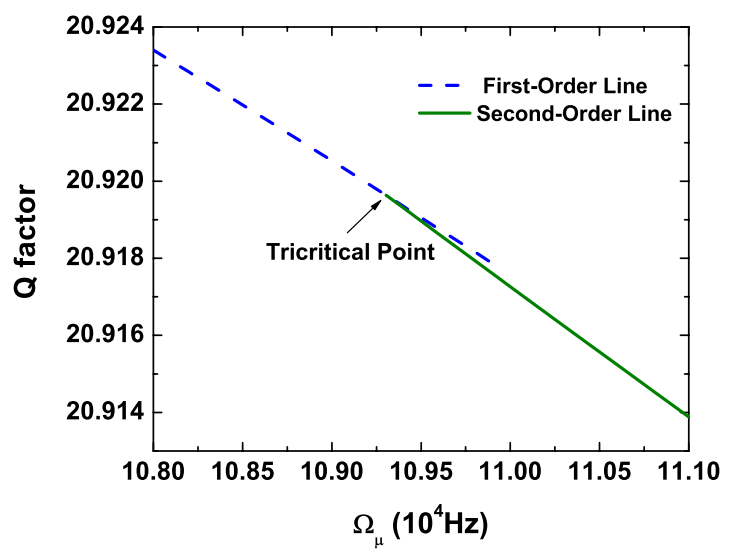

FIG. 3. (Color online) Phase diagram for the system. The firstorder phase transition line meets the second-order line at the tricritical point and goes beyond.

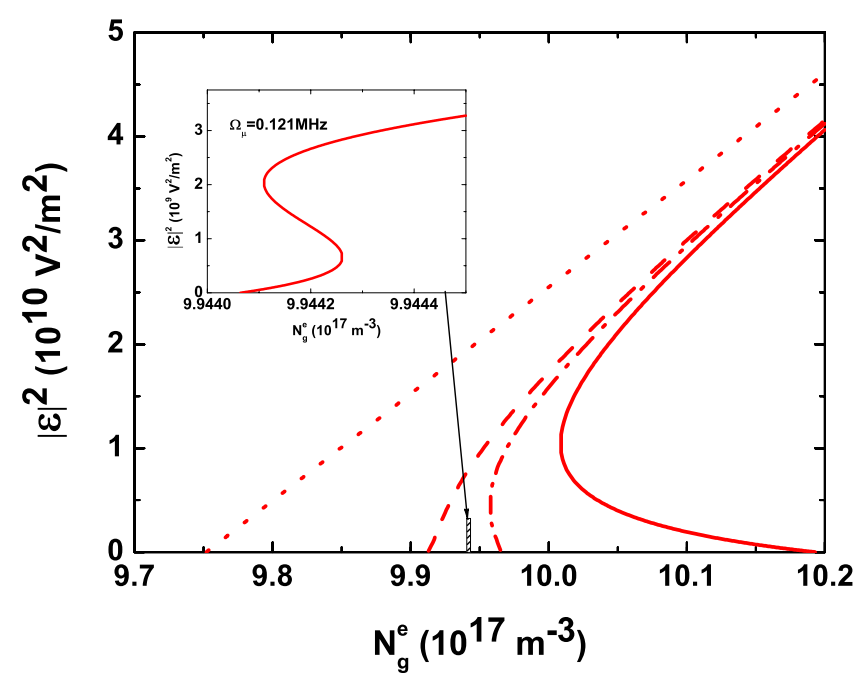

FIG. 4. (Color online) Intensity- $N_{g}^{e}$ curves for different drive Rabi frequencies. From right to left, $\Omega_{\mu}=0.011 \mathrm{MHz}, 0.11 \mathrm{MHz}$, $0.14 \mathrm{MHz}, 1.1 \mathrm{MHz}$. Similarly there is a switch between firstorder and second-order phase transitions. In the small window enlarged around the tricritical point, $\Omega_{\mu}=0.121 \mathrm{MHz}$ and there are three nonzero solutions. The other parameters are $\left|\wp_{a b}\right|$ $=10^{-29} \mathrm{~m} \mathrm{C}, \quad \lambda=1 \mu \mathrm{m}, \quad N_{a}^{e}=4.5 \times 10^{16} \mathrm{~m}^{-3}, \quad Q=27$, and $\gamma_{c b}$ $=1 \mathrm{kHz}$.

serve a first-order transition, or a second-order transition followed by a first-order transition, or a second-order transition.

We use the $Q$ factor as the variable to investigate the phase transition because it is the only parameter not in the expression of $C_{4}$. So we can compare the criterion $C_{4}=0$ to the numerical results. Without that purpose other parameters may also be used as the variable, for example, the effective number density of the gain medium. It is proportional to the discharge current if we use a gas discharge cell as the gain cell [18]. The numerical calculation shown in Fig. 4 is similar to Fig. 2. For a given drive Rabi frequency there could be one, two, or three solutions.

The latent heat in first-order phase transition is also an interesting topic [8]. At the critical point, the ClausiusClapeyron equation is

$$
L=T_{0}\left(V_{2}-V_{1}\right) \frac{d P}{d T_{0}}
$$

In our system, minus threshold population inversion corresponds to the critical temperature $T_{0}$, the drive field corresponds to the pressure $P$, and the laser field corresponds to the volume $V . \mathcal{E}_{1}$ and $\mathcal{E}_{2}$ can be solved from Eq. (15).

The LSA problem has also been investigated from the dispersive point of view. Mandel showed that if both cells contains two-level atoms, there could be new solutions corresponding to nonzero detuning because of the anomalous dispersion [26]. Lukin et al. considered the intracavity EIT and found a pronounced frequency pulling and cavitylinewidth narrowing [27]. Here we are mainly interested in the absorption property, so we take the detuning to be zero to avoid the pulling effect. 


\section{CONCLUSION}

In this paper, we investigate the effect of including an EIT cell as an absorber inside the laser cavity. By controlling the drive Rabi frequency, we can simulate the cases of laser with or without absorber and obtain phase transitions of both first order and second order. Around the tricritical point there could even be a second-order phase transition followed by a first-order one. These phenomena can be seen clearly from the phase diagram, in which the first-order phase transition line goes beyond its joint with the second-order line. The tricritical value determined by the criterion $C_{4}=0$ is close to the numerical result but not very accurate, because in this case the probe field is no longer small. Our calculation is based on $\Lambda$-type system. Other three-level systems like $\mathrm{V}$ type or $\Xi$ type should have a similar drive intensity controlled phase transition as well.

\section{ACKNOWLEDGMENT}

This research was partially supported by Qatar National Research Fund (QNRF).
[1] V. DeGiorgio and M. O. Scully, Phys. Rev. A 2, 1170 (1970).

[2] R. Graham and H. Haken, Z. Phys. 237, 31 (1970).

[3] H. Haken, Rev. Mod. Phys. 47, 67 (1975).

[4] M. Corti and V. DeGiorgio, Phys. Rev. Lett. 36, 1173 (1976).

[5] A. Gatti and L. Lugiato, Phys. Rev. A 52, 1675 (1995).

[6] A. P. Kazantsev, S. G. Rautian, and G. I. Surdutovich, Zh. Eksp. Teor. Fiz. 54, 1409 (1968) [Sov. Phys. JETP 27, 756 (1968)]; A. P. Kazantsev and G. I. Surdutovich, Zh. Eksp. Teor. Fiz. 56, 2001 (1969) [Sov. Phys. JETP 29, 1075 (1969)]; A. P. Kazantsev and G. I. Surdutovich, Zh. Eksp. Teor. Fiz. 58, 245 (1970) [Sov. Phys. JETP 31, 133 (1970)].

[7] R. Salomaa and S. Stenholm, Phys. Rev. A 8, 2695 (1973); R. Salomaa, J. Phys. A 7, 1094 (1974).

[8] J. F. Scott, M. Sargent III, and C. D. Cantrell, Opt. Commun. 15, 13 (1975).

[9] A. Baczynski, A. Kossakowski, and T. Marszalek, Z. Phys. B 23, 205 (1976); R. B. Schaefer and C. R. Willis, Phys. Rev. A 13, 1874 (1976).

[10] P. H. Lee, P. B. Schoefer, and W. B. Barker, Appl. Phys. Lett. 13, 373 (1968); H. M. Gibbs, S. L. McCall, and T. N. C. Venkatesan, Phys. Rev. Lett. 36, 1135 (1976).

[11] S. Ruschin and S. H. Bauer, Chem. Phys. Lett. 66, 100 (1979).
[12] J. W. Won, Opt. Lett. 8, 79 (1983).

[13] L. A. Lugiato, P. Mandel, S. T. Dembinski, and A. Kossakowski, Phys. Rev. A 18, 238 (1978).

[14] S. T. Dembinski, A. Kossakowski, L. A. Lugiato, and P. Mandel, Phys. Rev. A 18, 1145 (1978).

[15] P. Mandel and T. Erneux, Phys. Rev. A 30, 1893 (1984); T. Erneux and P. Mandel, ibid. 30, 1902 (1984).

[16] R. Roy, Phys. Rev. A 20, 2093 (1979).

[17] J. F. Scott, Opt. Commun. 15, 343 (1975).

[18] M. Mortazavi and S. Singh, Phys. Rev. Lett. 64, 741 (1990).

[19] P. Mandel, Z. Phys. B 33, 205 (1979).

[20] G. S. Agarwal and S. Dattagupta, Phys. Rev. A 26, 880 (1982)

[21] G. S. Agarwal, Phys. Rev. A 26, 888 (1982).

[22] P. Mandel and F.-K. Fang, Phys. Lett. 83A, 59 (1981).

[23] G. P. Agrawal, Phys. Rev. A 24, 1399 (1981).

[24] S. E. Harris, J. E. Field, and A. Imamoglu, Phys. Rev. Lett. 64, 1107 (1990).

[25] M. Mortazavi, F. Rawwagah, and S. Singh, Phys. Rev. A 65, 025803 (2002)

[26] P. Mandel, Phys. Lett. 83A, 207 (1981).

[27] M. D. Lukin, M. Fleischhauer, M. O. Scully, and V. L. Velichansky, Opt. Lett. 23, 295 (1998). 